\title{
Bootstrapping star position and baseline knowledge with uncalibrated SIM observations
}

Joseph Catanzarite, Ipek Basdogan, Mark H. Milman

Joseph Catanzarite, Ipek Basdogan, Mark H. Milman, "Bootstrapping star position and baseline knowledge with uncalibrated SIM observations," Proc. SPIE 4852, Interferometry in Space, (26 February 2003); doi: $10.1117 / 12.460855$

Event: Astronomical Telescopes and Instrumentation, 2002, Waikoloa, Hawai'i, United States 


\title{
Bootstrapping star position and baseline knowledge with uncalibrated SIM observations
}

\author{
J. Catanzarite ${ }^{* a}$, I. Basdogan ${ }^{\mathrm{b}}$ and M.H. Milman ${ }^{\mathrm{b}}$ \\ ${ }^{a}$ Interferometry Science Center, California Institute of Technology \\ Mail Stop 301-486, 4800 Oak Grove Drive, Pasadena, CA 91109, U.S.A. \\ bet Propulsion Laboratory, California Institute of Technology
}

\begin{abstract}
An external calibration technique for SIM $^{1,2,3}$ involves measurement of calibration stars whose positions must already be known to an accuracy of 2 milliarcseconds. We demonstrate a procedure that effectively "bootstraps" calibration star positions from an $a b$ initio catalog to the required accuracy by observing them with the uncalibrated SIM instrument.
\end{abstract}

Keywords: Space Interferometry Mission, SIM, calibration, astrometry

\section{INTRODUCTION}

The bootstrapping procedure involves SIM measurements of a semi-regular 10 by 10 grid of $10^{\text {th }}$ magnitude calibration stars in three different baseline positions. Two of the baselines are oriented orthogonal to each other and to the direction to the center of the calibration field; the third baseline is canted one degree away from either the first or second baseline toward the center of the calibration field.

We model the calibration stars as static points, without proper motion or parallax. We assume that the calibration star positions have apriori knowledge errors of 20 milliarcseconds. We allow errors of 2 arcseconds on the baseline orientations, as will be provided by the SIM attitude control system. We assume ${ }^{1}$ a 5 micron knowledge error on the absolute baseline length, and a 3 micron knowledge error on the bias offset constant. We assume Gaussian delay measurement errors of 250 picometers.

We generate an input catalog, with true and apriori positions of calibration stars, and apriori baselines. Using the input catalog, we generate simulated regularized SIM delay measurements and regularized baselines ${ }^{1}$. Finally we fit the delay measurements to a model equation for the parameters, including star positions and baseline orientations.

\section{INPUT AND TRUE CATALOGS}

For the input catalog we generated a 10 by 10 semi-regular grid of calibration stars (assumed to be $10^{\text {th }}$ magnitude) on a $15^{\circ}$ by $15^{\circ}$ square. The stars were then mapped onto the celestial sphere with the field centered on the North Celestial Pole, along the z-axis of a three-dimensional Cartesian coordinate system. The stars are observed using three baseline orientations: $b_{1}=\left[\begin{array}{lll}1 & 0 & 0\end{array}\right], b_{2}=\left[\begin{array}{lll}0 & 1 & 0\end{array}\right], b_{3}=R_{x}\left(1^{\circ}\right) b_{2}$

Note that $b_{3}$ is obtained by rotating $b_{2}$ by 1 degree about the y axis, thus canting the baseline toward the North Celestial Pole.

To generate the true catalog we perturb the input catalog positions randomly with 20 milliarcsecond rotations. Define basis vectors $t_{1}$ and $t_{2}$ in the tangent plane to the star vector: $t_{1}=(\mathrm{k} \times \mathrm{s}) / \mathrm{k} \times \mathrm{s} \mid$ along azimuthal direction (RA advance) where $\mathrm{k}=$ $\left[\begin{array}{lll}0 & 0 & 1\end{array}\right]=$ North Pole and $\mathrm{s}=$ apriori star vector; and $\mathrm{t}_{2}=\left(\mathrm{s} \times \mathrm{t}_{1}\right) / \mathrm{s} \times \mathrm{t}_{1} \mid$ along radial direction (DEC advance). Next, define a rotation perturbation vector $\omega_{\text {star }}$ in the star's tangent plane: $\omega_{\text {star }} \equiv \mathrm{t}_{1} \xi \cos \phi+\mathrm{t}_{2} \xi \sin \phi \equiv \omega_{1} \mathrm{t}_{1}+\omega_{2} \mathrm{t}_{2}$. Here $\phi$ is a randomly chosen angle between 0 and $2 \pi$, and $\xi=20 \operatorname{arcsec} \sim 10^{-7}$ radians. The true star vector is constructed as $s_{\text {true }}=\mathrm{s}+\omega_{\text {star }} \times \mathrm{s}$.

\footnotetext{
*jcat@huey.jpl.nasa.gov
} 


\section{SIMULATING SIM MEASUREMENTS}

The apriori and true star positions, together with the baseline vectors, are used to generate SIM delay measurements of the calibration stars using a reasonably sophisticated model ${ }^{1}$ of the SIM instrument. Quasi-instantaneous SIM delay measurements are modeled ${ }^{1,2,3}$ as $d_{\text {measured }}=\left\langle b_{\text {true }}, s_{\text {true }}\right\rangle+c+\eta$, where $b_{\text {true }}$ is the true science interferometer baseline vector, $\mathrm{s}_{\text {true }}$ is the true star vector, $\mathrm{c}$ is the bias offset constant, and $\eta$ is the Gaussian measurement noise.

\section{FORMULATING THE LINEAR LEAST-SQUARES PROBLEM}

Delays can be estimated from apriori knowledge as $d_{\text {estimated }}=\langle\mathrm{b}, \mathrm{s}\rangle$, where $\mathrm{b}=$ apriori baseline vector, $\mathrm{s}=$ apriori star vector. We define residuals between measured and estimated delays as $D \equiv d_{\text {measured }}-d_{\text {estimated }}$

We linearize the delay residuals to obtain a system of normal equations $\mathrm{D}=\mathbf{A} \cdot \mathrm{p}$

Here $\mathbf{A}$ is the design matrix, $\mathbf{A}_{\mathbf{i j}}=\partial \mathrm{D}_{\mathrm{i}} / \partial \mathrm{p}_{\mathrm{j}}$ where $\mathrm{p}_{\mathrm{j}}$ is correction vector for the $\mathrm{j}^{\text {th }}$ parameter, so that $\mathrm{p}_{\text {est }}=\mathrm{p}_{\text {apriori }}+\mathrm{p}$.

For 100 calibration stars there are 210 parameters to fit, comprising

- 2 star rotation angles for each of the 100 calibration stars

- 2 baseline vector angles for each of three baseline orientations.

- a bias offset parameter for each of the three baseline orientations

- a baseline length $|b|$.

Observations of 100 calibration stars, each measured in three different baseline orientations generate 300 delay measurements. So the system of normal equations is over-determined.

\section{SOLVING FOR THE STAR AND BASELINE PARAMETERS}

We first attempt to solve the linear least-squares problem using Singular Value Decomposition ${ }^{5}$. SVD gives an estimate of the parameter corrections vector $\mathrm{p}=\mathrm{A}^{\dagger} \mathrm{D}$, where $\mathrm{A}^{\dagger}$ is the pseudoinverse ${ }^{6}$ of $\mathrm{A}$.

The least-squares estimator minimizes the sum of squares of the differences $D-D_{\text {est }}$, where $D_{\text {est }}=\mathbf{A} \cdot \mathbf{p}$ are the delay residuals using the estimated parameter corrections vector.

We found that the SVD solution does not improve the star positions. The reason is that baseline length is degenerate with $\omega_{1}$ star rotation parameters. Recall that the $\omega_{1}$ are rotations along the radial direction from zenith.

An alternative approach is the Gauss-Markov estimator ${ }^{4}$ which gives $p=\left(A^{T} Q^{-1} A+R^{-1}\right) A^{T} Q^{-1} D$. Here, $\mathrm{Q}$ is the noise matrix for the measurements, and $\mathrm{R}$ is the noise matrix for the parameters.

The Gauss-Markov estimator uses a Bayesian approach, incorporating apriori knowledge of errors in measurements and in parameter estimates; it minimizes the sum of the variances of the fitted parameter residuals.

Because it makes use of apriori information, the Gauss-Markov estimator achieves good estimates for baseline length and bias offset parameters. The resulting star position estimates are accurate to better than 2 milliarcseconds, and baseline orientation estimates are accurate to about 25 milliarcseconds. The caveats here are: we may not know the appropriate measurement and parameter errors well enough; also the parameter errors could be systematic and not random, as we have assumed. 


\section{RESULTS WITH THE GAUSS-MARKOV ESTIMATOR}

We determined fit residuals for baseline and star rotation parameters $\omega_{\mathrm{bi} 1}, \omega_{\mathrm{bi} 2}, \omega_{\mathrm{si} 1}, \omega_{\mathrm{si} 2}$, and baseline length $|\mathrm{b}|$.

- RMS of star rotation parameter fit residuals was less than $7.5 \times 10^{-09}$ or $\sim 1.5$ milliarcseconds

- Errors on the baseline orientations were less than $1.2 \times 10^{-07}$ radians, or $\sim 25$ milliarcseconds

- Residual of estimated baseline length is -0.05 microns.

As a check, we verified that the RMS fit residuals for the parameters were consistent with the parameter accuracies predicted by the covariance matrix.

Figure 1 shows the residuals in distances between pairs of calibration stars as a function of separation.

Figure 2 shows the residuals from the covariance matrix, for the radial and azimuthal rotation parameters, for each star. Star indices were generated with a 10-by-10 raster scan, which is the reason for the spatially periodic structures seen in the plots.

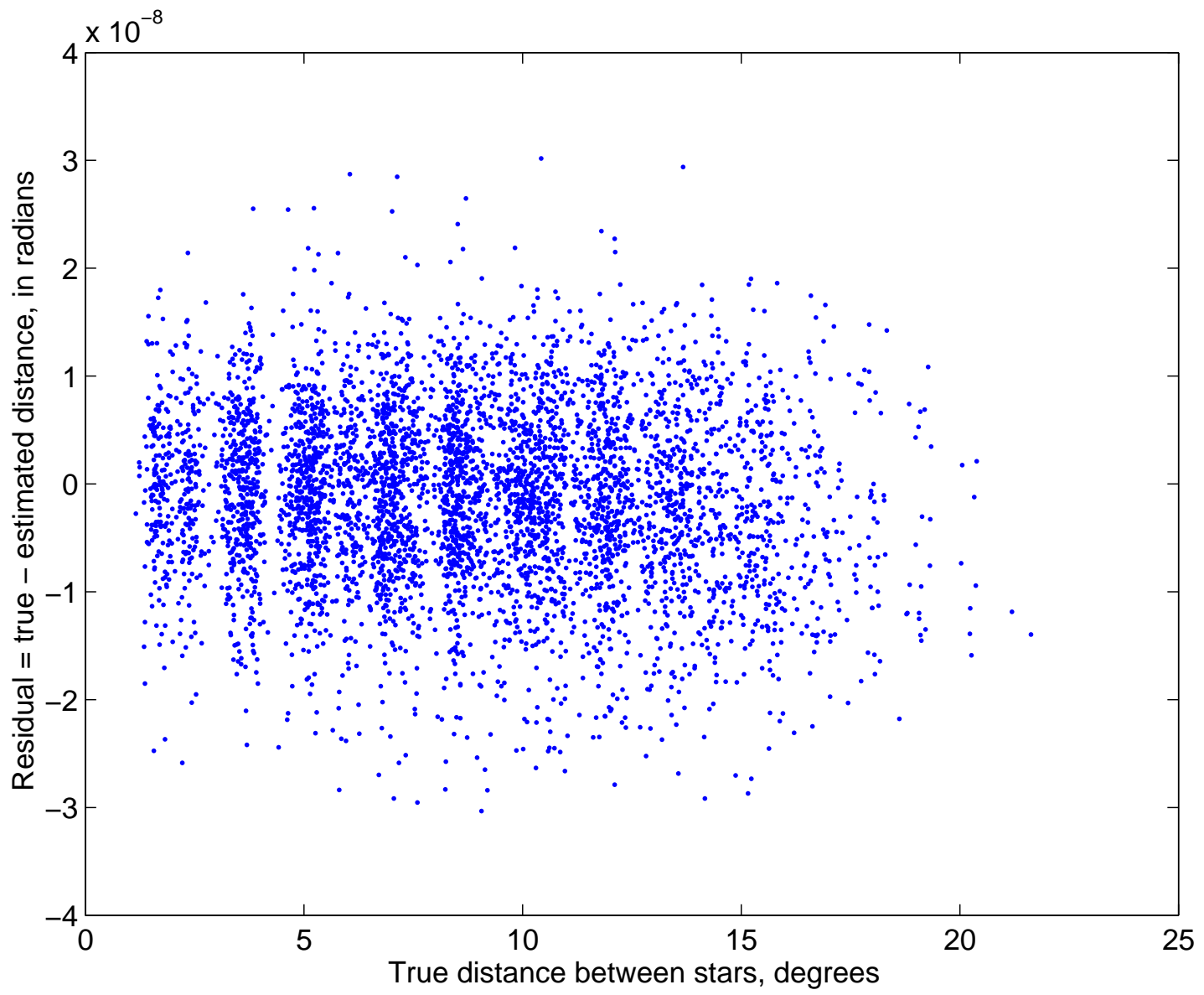

Figure 1. Residuals in distances between pairs of stars versus separation. 

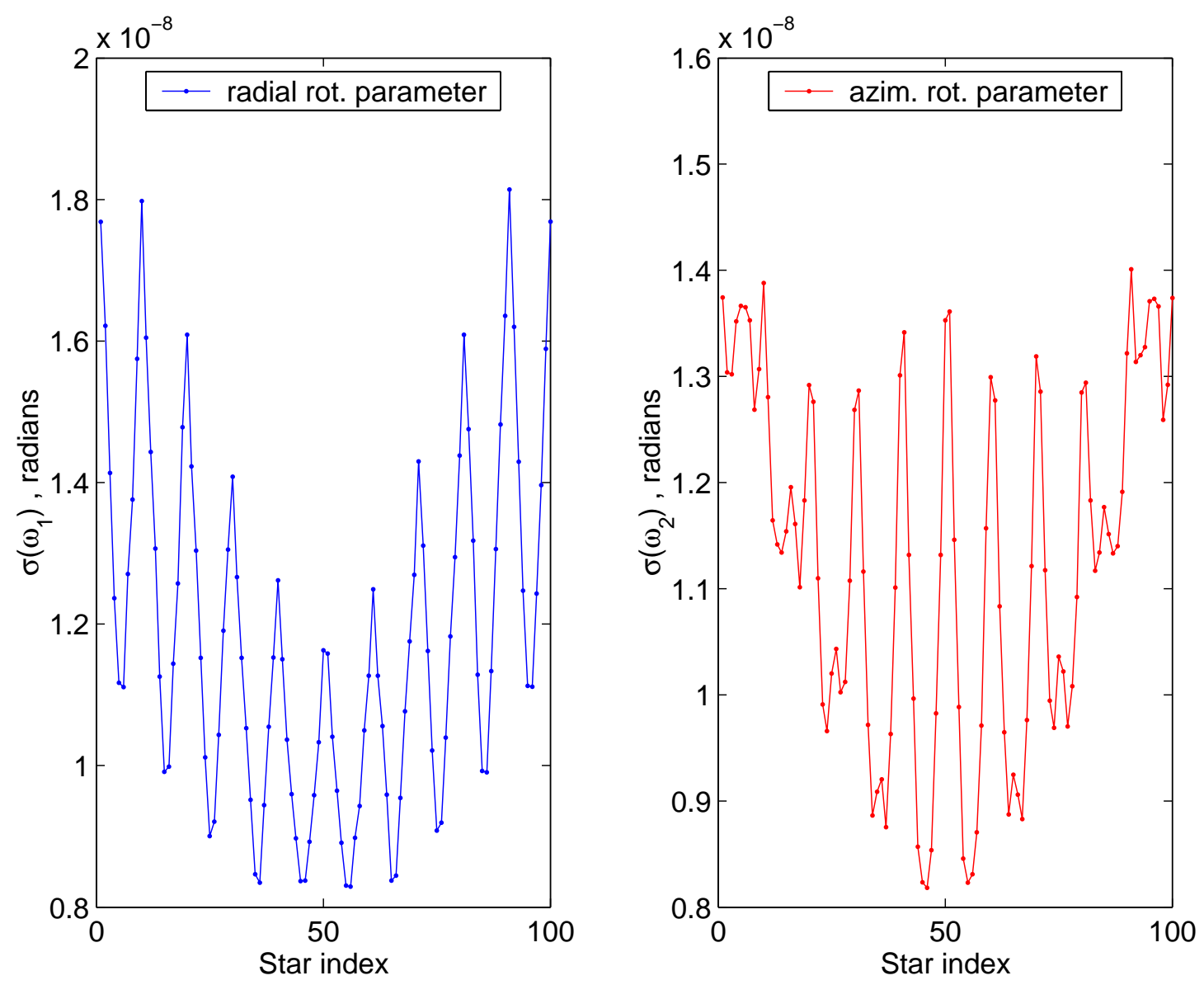

Figure 2. Star parameter accuracies for Gauss-Markov estimator solution, from covariance matrix

\section{CONCLUSION}

We have demonstrated a technique to 'bootstrap' knowledge of calibration star positions from ab initio accuracy of 20 milliarcseconds to better than 2 milliarcseconds, and baseline orientations from $a b$ initio accuracy of 2 arcseconds to about 25 milliarcseconds. These improved star positions and baselines provide a sufficient level of apriori knowledge to operate the external calibration technique ${ }^{3}$. Further investigations are needed to determine the effects of systematic 'zonal errors' in the input catalog.

\section{ACKNOWLEDGEMENTS}

The research described in this paper was carried out at the Jet Propulsion Laboratory, California Institute of Technology, under a contract with the National Aeronautics and Space Administration.

\section{REFERENCES}

1. L. Sievers, M.H. Milman, S.B. Shaklan, R.P. Korechoff, J. Catanzarite, I. Basdogan, M.V. Papalexandris, R. Swartz, "Overview of SIM calibration strategies", SPIE Astronomical Telescopes and Instrumentation Conference Proceedings, August 2002, paper [4852-06]. 
2. S.B. Shaklan, M.H. Milman, J. Catanzarite, I. Basdogan, M.V. Papalexandris, L. Sievers, R. Swartz, "Overview of SIM external calibration", SPIE Astronomical Telescopes and Instrumentation Conference Proceedings, August 2002, paper [4852-09].

3. M. V. Papalexandris, M.H. Milman, S.B. Shaklan, "Description of a proposed on-orbit calibration procedure for SIM based on spacecraft maneuver", SPIE Astronomical Telescopes and Instrumentation Conference Proceedings, August 2002, paper [4852-70].

4. D. G. Luenberger, Optimization by Vector Space Methods, Wiley 1969

5. W.H. Press, S.A. Teukolsky, W.T. Vetterling, and B. P. Flannery, Numerical Recipes in C, $2^{\text {nd }}$ edition, Wiley 1992

6. C.L. Lawson and R.J. Hanson, Solving Least Squares Problems, Prentice-Hall 1974 